\title{
From point defects to dislocation loops: a comprehensive TCAD model for self-interstitial defects in silicon
}

\author{
Ignacio Martin-Bragado and Ibrahim Avci \\ Synopsys Inc. \\ Mountain View, CA 94043, USA \\ Email: nacho@synopsys.com
}

\author{
Nikolas Zographos \\ Synopsys Switzerland LLC \\ 8050 Zurich, Switzerland
}

\author{
Pedro Castrillo and Martin Jaraiz \\ Departamento de Electronica \\ Universidad de Valladolid \\ 47011 Valladolid, Spain
}

\begin{abstract}
An atomistic model for self-interstitial extended defects is presented in this work. Using a limited set of assumptions about the shape and emission frequency of extended defects, and taking as parameters the interstitial binding energies of extended defects versus their size, this model is able to predict a wide variety of experimental results. The model accounts for the whole extended defect evolution, from the initial small irregular clusters to the $\{311\}$ defects and to the more stable dislocation loops. The model predicts the extended defect dissolution, supersaturation and defect size evolution with time, and it takes into account the thermally activated transformation of $\{311\}$ defects into dislocation. The model is also used to explore a two-phase exponential decay observed in the dissolution of $\{311\}$ defects.
\end{abstract}

\section{INTRODUCTION}

Current technology uses ion implantation as the main process to introduce dopants in silicon. Inherent to this process is the creation of a high amount of point defects, leading to the formation of different defect agglomerates. Their subsequent dissolution during annealing generates a point defect supersaturation that affects the diffusion of the implanted dopants. A thorough understanding of the dissolution kinetics of these defects is needed in order to correctly predict and control the final dopant profile in the deep sub-micron regime. In particular, extra self-interstitials (I) released both from $\{311\}$ rodlike defects [1] and small clusters [2] cause the Transient Enhanced Diffusion (TED) of commonly used dopants. Four types of selfinterstitial extended defects have been detected experimentally in silicon: [3] small irregular clusters, $\{311\}$ defects, and faulted and perfect dislocation loops (DLs). All of these defects are of extrinsic character, i.e. they are formed with extra $\mathrm{Si}$ atoms precipitated as clusters. A study about the smaller precursor clusters that nucleate and grow into $\{311\}$ 's was reported by Cowern et al. [4]. Based on experimental observations [5], the unfaulting of the $\{311\}$ defects is the source of the subthreshold DLs in non-amorphized ion-implanted silicon, i.e. the $\{311\}$ defects can either dissolve or unfault into loops.

The total TED depends mainly on the amount of excess interstitials and on the depth at which the defects are formed. This interstitial supersaturation is related to the energetics of the interstitial defects and defects present in the sample [4], [6]. Consequently to correctly account for TED for incomplete anneals, process simulators have to implement predictive models for the evolution of the small clusters, $\{311\}$ defects and DLs. In particular, the formation of DLs decreases the supersaturation by several orders of magnitude and it will severely affect the dopant profiles after the incomplete annealing. In consequence, the modelling and accurate prediction of the transition from $\{311\}$ defects to DLs is imperative.

A considerable effort, using both continuum [4], [7]-[13] and atomistic [14]-[16] approaches, has been devoted to the understanding of the physical mechanisms that control the nucleation, growth and dissolution of such defects. The continuum method however, is limited by the number of equations that can be solved without run- ning into prohibitive CPU demands and/or convergence instabilities. Moreover, it uses some simplifying assumptions about the capture volume, and it makes a continuum treatment of the discrete extended defects.

In this work, we have developed a comprehensive extended defect model which accounts for the whole defect evolution: the point defects nucleate into small clusters which will transform into $\{311\}$ defects which finally can become DLs. The model has been implemented in an atomistic kinetic Monte Carlo (kMC) simulator [17] using a single set of parameters to explain all the different simulation conditions.

\section{PHYSICAL MODEL}

In our model $I \mathrm{~s}$ and vacancies are represented as points in a $3 \mathrm{D}$ simulation domain, and they are given random jumps at a rate derived from their diffusivities. They can interact with other particles which are found within their capture radius, leading to cluster formation or recombination. The jump distance and the capture radius is always assumed to be the second neighbor's distance in the silicon lattice.

\section{A. Shape}

Our model assumes the shape of interstitial clusters with size $n<15$ interstitials to be irregular. For bigger sizes we rearrange them into the $\{311\}$ defects and/or faulted DLs according to the crystalline geometry data. The experimental transition size between small clusters and $\{311\}$ defects is not well known, and the literature establishes a size of $n=10$ as a minimum [4], [12] and $n=40$ as a maximum [11].

We assume that irregular clusters retain captured point defects at their arrival position. This assumption leads naturally to a roughly spherical shape. On the other hand $\{311\}$ defects are modeled as parallel stripes (rows) of interstitials lying in one of the twelve orientations, randomly chosen, of a $\{311\}$ plane. We model the $\{311\}$ defect shape, following the experimental data [18], as $N_{\text {row }}$ rows of Is lying on a $<01 \overline{1}>$ line with a distance of $a / \sqrt{2}$ between $I \mathrm{~s}$ in the same line and $N_{\text {col }}$ columns keeping a distance of $a \sqrt{22} / 4$ between them, being $a=0.543 \mathrm{~nm}$ the silicon lattice parameter. We assume that the ratio between length $(L)$ and width $(W)$ is given by [9] $W \approx \sqrt{C L}$, with $C=0.5 \mathrm{~nm}$. Consequently, the length of the defects is $L \approx 0.5 n^{2 / 3} \mathrm{~nm}, n$ being the defect size (number of $I \mathrm{~s}$ ). In our model, $\{311\}$ defects capture any point defect jumping into the capture volume of the particles belonging to the defect. After the capture, the number of $N_{\text {col }}$ and $N_{\text {row }}$ is recalculated, with a small hysteresis to prevent $\{311\}$ defect reshape due to the emission and capture of the same particle.

The transformation of $\{311\}$ defects into DLs depends on the size of the $\{311\}$ defects and on the temperature. DL are expected to be more stable than $\{311\}$ defects beyond a size of about $\approx 350$ atoms. 


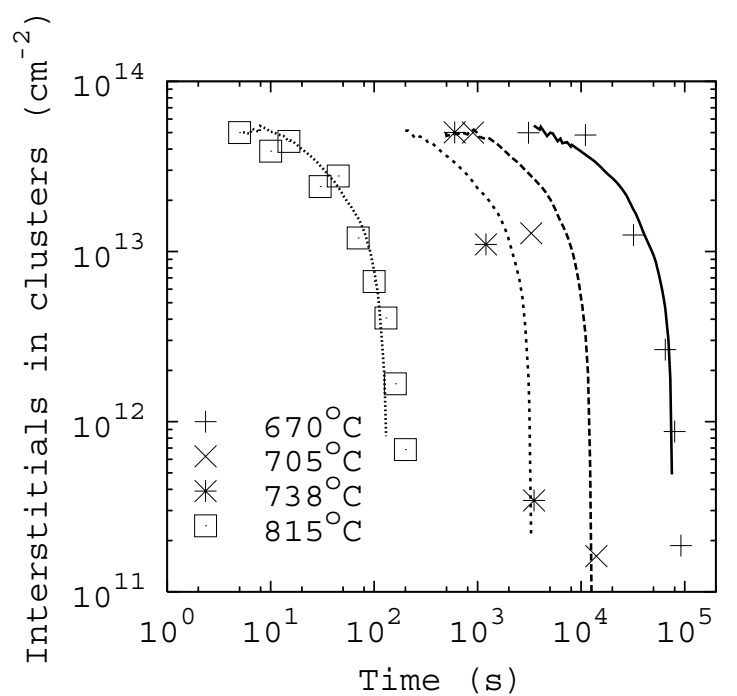

Fig. 1. Self-interstitial concentration in clusters as a function of annealing time and temperature, after a $40 \mathrm{keV}, 5 \times 10^{13} \mathrm{~cm}^{-2}$, Si implant. Symbols are experimental data taken from Ref. [1]. Lines are simulation results.

However, $\{311\}$ defects of much larger sizes have been observed [1], [5], [11], [18]. For a $\{311\}$ defect population distribution (at a given temperature), only the large $\{311\}$ defects are observed to transform into DLs. Moreover, at low temperature, very large $\{311\}$ defects can be observed [18], while at higher temperatures smaller $\{311\}$ defects are observed to transform into DLs [5]. This suggests a thermally activated transition, whereby the probability is a strong function of the size of $\{311\}$ defects. However, a very similar behavior can be obtained with a much simpler model. In the simple model we have used, there is a critical size for transformation of $\{311\}$ defects into DLs $\left(n_{\mathrm{min}}^{\text {loop }}\right)$, and it is a function of temperature. When a $\{311\}$ reaches $n_{\mathrm{min}}^{\text {loop }}$, it will immediately transform into a DL. $n_{\min }^{\text {loop }}$ is assume to follow an Arrhenius plot:

$$
n_{\text {min }}^{\text {loop }}=N^{\text {loop }} \exp \left(E_{\{311\} \rightarrow \text { loop }} / k_{B} T\right),
$$

$E_{\{311\} \rightarrow \text { loop }}$ being the activation energy for the transition size and $N^{\text {loop }}$ the prefactor. The DL formation energy has to be smaller than the $\{311\}$ formation energy at the threshold size $\left(n_{\min }^{\text {loop }}\right)$, otherwise the threshold is taken as the size where both energies are equal. We find $n_{\mathrm{min}}^{\text {loop }}=0.68 \mathrm{eV}$ and $N^{\text {loop }}=1.6$ atoms. DLs are assumed to be always unfaulted discs lying on a $\{111\}$ plane. Their interstitial area density is taken as $d_{\{111\}}=8 /\left(\sqrt{(3)} a^{2}\right)$, the atomic density of $\{111\}$ planes, and consequently its radius will be $r_{\text {loop }}=\sqrt{\pi n / d_{\{111\}}}$.

\section{B. Emission and binding energies}

Extended defects are allowed to emit their constituent particles. The rates of these processes are determined by specific activation energies derived from molecular dynamics, first principles calculations and experiments. In particular, the activation energy for $I$ emission from an $I$ extended defect of size $n$ is calculated as the sum of the binding energy plus the $I$ migration energy (taken as $0.7 \mathrm{eV}$ ). The emission prefactor is assumed to be proportional to the cluster surface, in correspondence with the capture process. Small clusters emit particles from the position where they were captured, whereas $\{311\}$ defects and loops take the last captured particle from the periphery, and emit it from a random position on the surface of the defect, to conserve microscopic reversibility with the capture process.

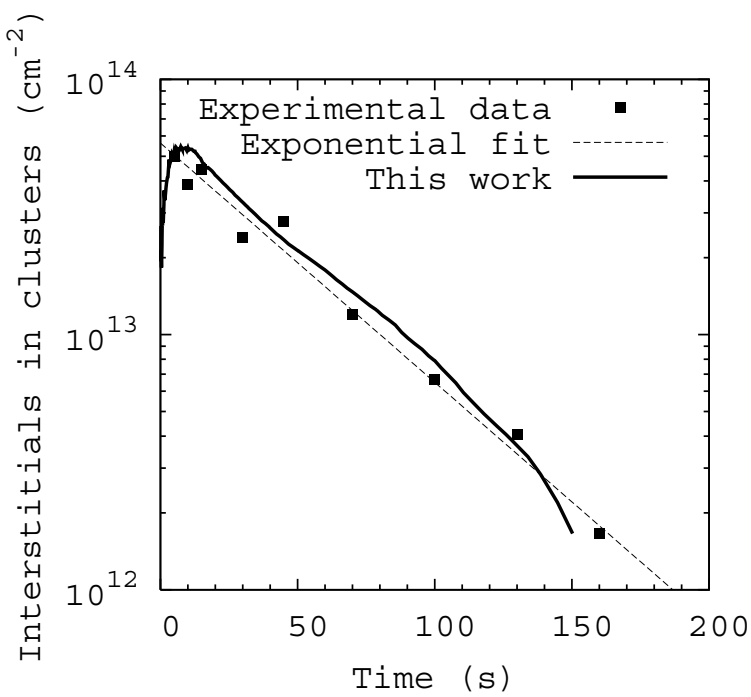

Fig. 2. Comparison between the experimental trapped interstitial dose at $815^{\circ} \mathrm{C}$ (symbols, Ref. [1]), and the simulated one (solid line). The plot has a semi logarithmic scale to reveal the exponential decay with time. An exponential fit is also shown as a reference (dashed line).

Finally, the binding energy of a size $n$ loop is calculated as

$$
E_{b}^{\mathrm{loop}}(n)=E_{f I}+E_{f}^{\mathrm{loop}}(n-1)-E_{f}^{\mathrm{loop}}(n),
$$

where the DLs formation energy, $E_{f}^{\text {loop }}$, is taken from Ref. [19] and the formation energy of a free interstitial $\left(E_{f I}\right)$ is assumed to be $4.0 \mathrm{eV}$.

\section{RESULTS AND DISCUSSION}

\section{A. Extended defect dissolution}

The self-interstitial concentration in $\{311\}$ defects as a function of annealing time is represented in Fig. 1. It corresponds to a $5 \times 10^{13} \mathrm{~cm}^{-2}, 40 \mathrm{keV} \mathrm{Si}$ implant annealed at 670, 705, 738 and $815^{\circ} \mathrm{C}$. Experimental data (symbols) have been taken from Ref. [1]. The simulations (lines) are done in a $150 \times 150 \mathrm{~nm}^{2}$ simulation domain. As it can be seen, the agreement is very good. Fig. 2 represents the $815^{\circ} \mathrm{C}$ simulation in a semi-log scale together with experimental data [20] to reveal the exponential decay with time. In this case, the simulation data is not statistically representative for concentrations below $\approx 5 \times 10^{12} \mathrm{~cm}^{-2}$, because there are just a few $\{311\}$ defects remaining in the whole simulation domain. The exponential behavior is obtained due to the binding energy dependency with size for big clusters. A constant value would lead to a linear, instead of exponential, decay with time [6].

For the final defect dissolution stages, Ref. [20] suggested that the "decay curves are not purely exponential, but are likely to be more convex instead". The same idea is explored in Ref. [11], [14], which suggests the presence of two different dissolution regimes, with a transition between both, when the average defect-to-defect distance becomes larger than the defect-to-surface distance. To properly test the two-phase dissolution concept, and to analyze quantitatively the transition between the two regimes, we have run a $\times 200 \times 200 \mathrm{~nm}^{2}$ simulation, using a shallower Si implant centered at $\bar{x}=30 \mathrm{~nm}$ which would lead to a transition at a higher concentration, and then better statistics. Fig. 3 shows the trapped interstitial concentration evolution from that simulation. Two exponential lines have been fitted to show the two different dissolution regimes. The first one, when the average distance between defects is smaller than to the surface, 


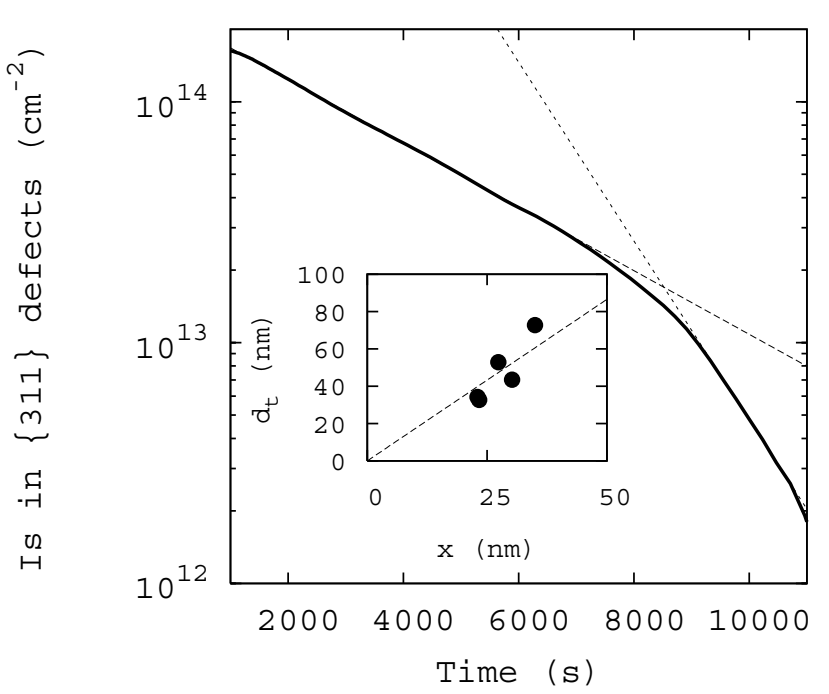

Fig. 3. Simulation exhibiting a two-phase $\{311\}$ defect dissolution. An interstitial profile, centered at $\bar{x}=30 \mathrm{~nm}$ with a peak concentration of $\approx 3 \times 10^{20}$ have been annealed at $700^{\circ} \mathrm{C}$. Two different exponential phases can be seen in each dissolution. The first regime accounts for defect to defect interactions, while the second one is related with the defect to surface recombinations. Inset: Dependency of the distance threshold with the defect average distance to the surface (see text).

$\left(1 \times 10^{3}\right.$ to $\left.6 \times 10^{3} \mathrm{~s}\right)$, and the second one, $\left(9 \times 10^{3}\right.$ to $\left.1.1 \times 10^{4} \mathrm{~s}\right)$, when the surface is closer than the nearest defect. From the Fig. 3 it can be seen that the threshold between both corresponds to a concentration of about $1.4 \times 10^{13} \mathrm{~cm}^{-2}$. A simple estimate of the threshold concentration can be done assuming that it occurs when the average defect distance to the surface $(\bar{x})$ equals the average distance between defects $d=\sqrt{\bar{N} /[I]}, \bar{N}$ being the average defect size. We can compute the threshold distance as $d_{t}=k \bar{x}, k$ being a factor to correlate the defect-to-defect distance with the defect-to-surface distance, expected to be between 1 and 2. This assumption gives a threshold trapped interstitial concentration of

$$
[I]_{t}=\frac{\bar{N}}{(k \bar{x})^{2}} .
$$

We extract $\bar{N}=260$ from the previous simulation which gives $k \approx 1.4$. We confirm this idea simulating the same profile shifted by different displacements, measuring $\bar{N}$ and $\bar{x}$ and plotting in Fig. 3 (inset) the dependency between the $d_{t}$ and $\bar{x}$. We find $k \approx 1.7$, in agreement with the intuition of $k \in[1,2]$. The threshold distance lets us establish different regimes during the extended defect dissolution. For $d<d_{t}$ the defects are close to each other, maintaining a conservative Ostwald ripening [14]. For $d>d_{t}$ the surface is closer than other extended defects, and the defects will dissolve fast through interaction with the surface, in a non conservative Ostwald ripening. We should remark the discrete character of this effect, that cannot be simulated with $1 \mathrm{D}$ process simulators.

\section{B. Interstitial supersaturation}

Predictive interstitial supersaturation models are needed to correctly simulate Transient Enhanced Diffusion (TED) of boron and other dopants. The supersaturation depends on the ripening of small clusters and is regulated by the surface. Our model correctly predicts the supersaturation as a function of time for different annealing temperatures. As a consequence, the agreement between the experimental

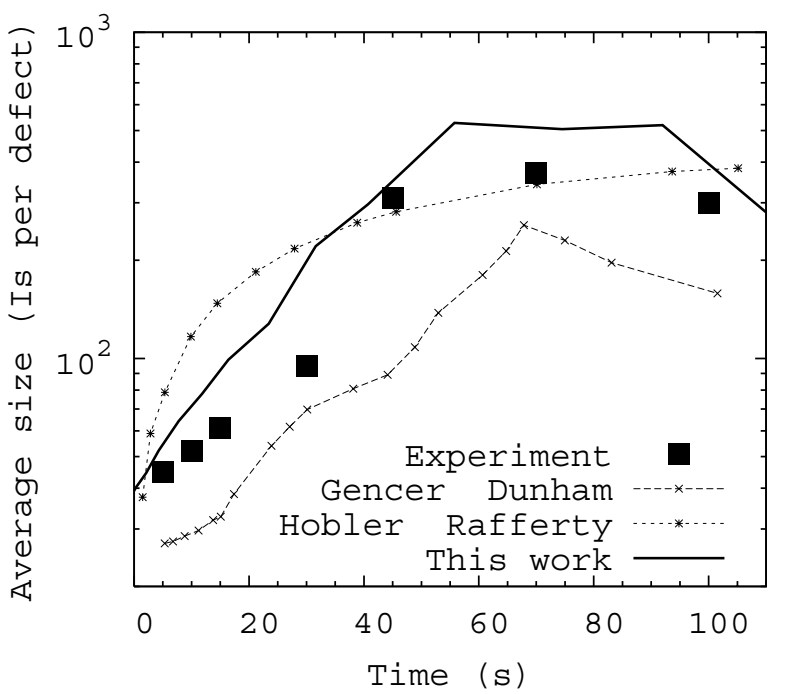

Fig. 4. Self-interstitial cluster average size evolution with annealing time. Symbols are the experimental results [1] from a $40 \mathrm{keV} 5 \times 10^{13} \mathrm{Si}$ implant and subsequent annealing at $815^{\circ} \mathrm{C}$. Different domain sizes have been used in the simulations, showing the need for a surface bigger than $80 \times 80 \mathrm{~nm}^{2}$ to correctly predict the average size for long annealing times.

data [4] and simulations [15] is excellent.

\section{Extended defects size evolution}

Figure 4 shows the time evolution of the $I$-cluster average size at $815^{\circ} \mathrm{C}$. The experimental data (symbols) are taken from Ref. [1], corrected using the square-root law of Ref. [9]. The lines correspond to the model presented here and to other models from the literature. The results presented in this work have been simulated using a $150 \times 150 \mathrm{~nm}^{2}$ simulation cell. For lateral sizes smaller than $k \bar{x}$ the simulations do not converge to the correct sizes. The good agreement of our model for small sizes (in comparison with Ref. [9]) is due to the use of oscillating energies, the presence of a stable cluster at size 8 retains the growth of the system in the first few seconds. The fit of Fig. 4 is going to be slightly dependent on the assumption about the ratio between $W$ and $L$.

Another study is presented in Fig. 5 showing a $40 \mathrm{keV}, 10^{14} \mathrm{~cm}^{-2}$ Si implant annealed at $750{ }^{\circ} \mathrm{C}$. Experimental data (symbols), showing the maximum and average size, and the standard deviation, were taken from Ref. [21], the simulated ones (lines) were obtained with a $100 \times 100 \mathrm{~nm}^{2}$ surface.

The ability to correctly reproduce experimental extended defect sizes is important to have physically-based predictive models and it is even more relevant when the transformation from $\{311\}$ defects to DLs is directly modelled as a function of the $\{311\}$ size.

\section{Transformation of $\{311\}$ defects into DLs}

Figure 6 shows the trapped interstitial concentration as a function of annealing time for both $\{311\}$ defects and DLs for a $100 \mathrm{keV}$, $2 \times 10^{14} \mathrm{~cm}^{-2} \mathrm{Si}$ implant during annealing at $800^{\circ} \mathrm{C}$. Symbols are experimental points taken from Ref. [5], lines are simulation results. The simulated data has been multiplied by a factor $1 / 3$ to take into account that in the experiment the total number of atoms $(\{113\}+$ loops) is somehow low with respect to the implanted dose $\left(2 \times 10^{14}\right)$. Nevertheless, the qualitative agreement validates the $\{311\}$ to loops transition model. 


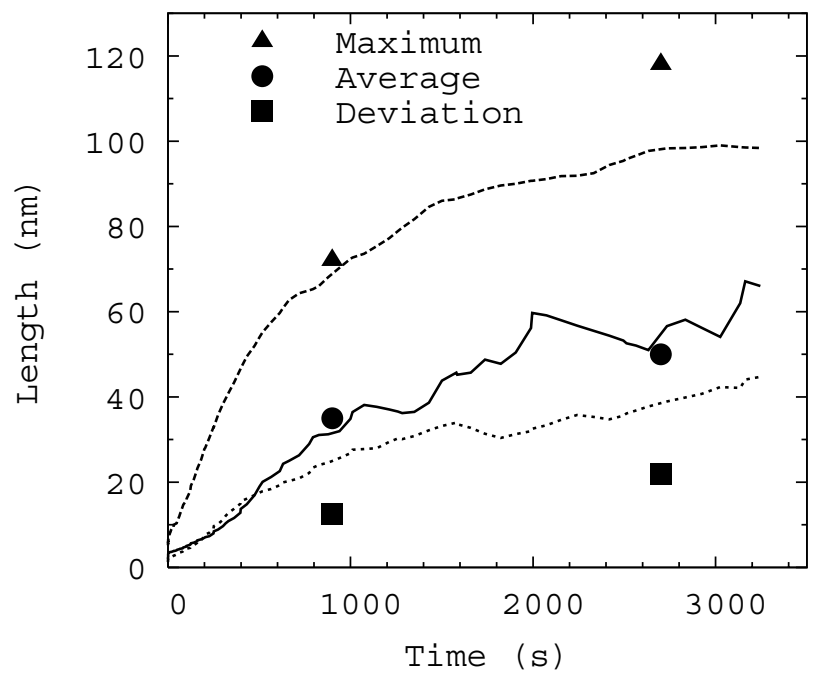

Fig. 5. Plot of the average, maximum size and standard deviation of the defect length as a function of anneal time at $750^{\circ} \mathrm{C}$ after a $40 \mathrm{keV}, 10^{14} \mathrm{~cm}^{-2} \mathrm{Si}$ implant. Symbols: experimental data (Ref. [21]). Lines: Simulations.

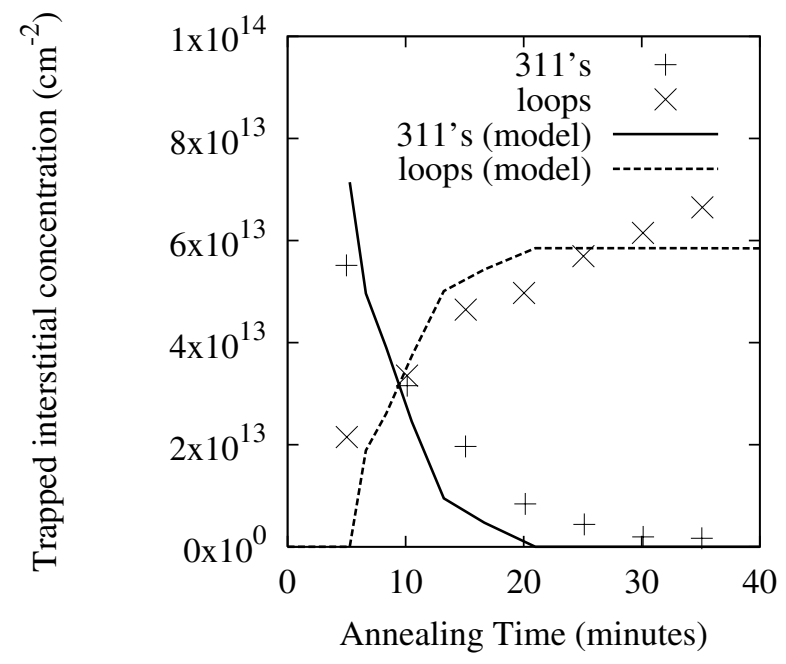

Fig. 6. Trapped interstitial concentration as a function of annealing time for both $\{311\}$ defects and DLs for a $100 \mathrm{keV}, 2 \times 10^{14} \mathrm{~cm}^{-2} \mathrm{Si}$ implant after annealing at $800^{\circ} \mathrm{C}$. Symbols are experimental points taken from Ref. [5], lines are simulation results.

\section{CONCLUSions}

In this work we present a comprehensive modelling framework for self interstitials defects in silicon that is able to reproduce a wide compilation of different extended-defect experiments using the same parameter set. The model, which has been implemented in an atomistic kinetic Monte Carlo simulator, includes small irregular clusters, $\{311\}$ rod-like defects and faulted DLs. The extended defect shapes are built in agreement with experimental crystallographic data. Thus, the appropriate emission/capture ratio can be reached since the emission depends on the defect binding energy, and the capture on the extended defect geometry.

Several experimental data is correctly predicted with the presented model: (i) the $\{311\}$ defect dissolution at different temperatures, (ii) the interstitial supersaturation evolution with time, (iii) the maximum and average defect-size time evolution and (iv) the $\{311\}$ to DLs transition. The model has also been used to confirm the presence and quantitatively analyze the transition between two exponential regimes during the $\{311\}$ defect dissolution.

\section{REFERENCES}

[1] D. J. Eaglesham, P. A. Stolk, H. J. Gossmann, and J. M. Poate, "Implantation and transient b diffusion in si: The source of the interstitials," Appl. Phys. Lett., vol. 65, no. 18, p. 2305, 1994.

[2] L. H. Zhang, K. S. Jones, P. H. Chi, and D. S. Simons, "Transient enhanced diffusion without $\{311\}$ defects in low energy $\mathrm{b}^{+}$-implanted silicon," Appl. Phys. Lett., vol. 67, pp. 2025-2027, 1995.

[3] A. Claverie, B. C. ad Gerard Ben Assayag, C. Bonafos, F. Cristiano, M. Omri, and B. de Mauduit, "Thermal evolution of extended defects in implanted si: impact on dopant diffusion," Mat. Sci. Semicon. Proc., vol. 3, pp. 269-277, 2000.

[4] N. E. B. Cowern, G. Mannino, P. A. Stolk, F. Roozeboom, H. G. A. Huizing, J. G. M. van Berkum, F. Cristiano, A. Claverie, and M. Jaraiz, "Energetics of self-interstitial clusters in Si," Phys. Rev. Lett., vol. 82, no. 22, pp. 4460-4463, 51999.

[5] J. Li and K. S. Jones, " $\{311\}$ defects in silicon: The source of the loops," Appl. Phys. Lett., vol. 73, no. 25, p. 3748, 1998.

[6] C. Rafferty, G. Gilmer, M. Jaraiz, D. Eaglesham, and H. Gossmann, "Simulation of cluster evaporation and transient enhanced diffusion in silicon,” Appl. Phys. Lett., vol. 68, no. 17, p. 2395, 1996.

[7] A. H. Gencer and S. T. Dunham, "A predictive model for transient enhanced diffusion based on evlution of $\{311\}$ defects," J. Appl. Phys., vol. 81, no. 2, pp. 631-36, January 1997.

[8] C. Bonafos, D. Mathiot, and A. Claverie, "Ostwald ripening of end-ofrange defects in silicon," J. Appl. Phys., vol. 83, no. 6, p. 3008, 1998.

[9] G. Hobler and C. S. Rafferty, "Modeling of $\{311\}$ defects," in Si Front End Processing, vol. 568. Materials Research Society, 1999, pp. 123134.

[10] I. Avci and M. E. Law, "Modeling dislocation loop nucleation and evolution in germanium, arsenic and boron implanted silicon," in Mat. Res. Soc. Symp. Proc., vol. 717, 2002, p. C5.9.1.

[11] F. Cristiano, N. Cherkashin, X. Hebras, P. Calvo, Y. Lamrani, E. Scheid, B. de Mauduit, B. Colombeau, W. Lerch, S. Paul, and A. Claverie, "Ion beam induced defects in crystalline silicon," Nucl. Instrum. Meth. B., vol. 216, pp. 46-56, 2004.

[12] C. J. Ortiz, F. Cristiano, B. Colombeau, A. Claverie, and N. E. B. Cowern, "Modeling of extrinsic extended defect evolution in ion-implanted silicon upon thermal annealing," Mat. Sci. Eng. B, 2004.

[13] N. Zographos, C. Zechner, and I. Avci, "Efficient TCAD model for the evolution of interstitial clusters, 311 defects, and dislocations loops in silicon," 2007, to be published in Mat. Res. Soc. Symp. Proc.

[14] M. Aboy, L. Pelaz, L. A. Marques, L. Enriquez, and J. Barbolla, "Atomistic analysis of defect evolution and transient enhanced diffusion in silicon," J. Appl. Phys., vol. 94, no. 2, pp. 1013-8, 2003.

[15] I. Martin-Bragado, S. Tian, M. Johnson, P. Castrillo, R. Pinacho, J. Rubio, and M. Jaraiz, "Modelling charge defects, dopant diffusion and activation mechanisms for TCAD simulations using kinetic monte carlo," Nucl. Instr. Meth. B, vol. 253, pp. 63-67, 2006.

[16] I. Martin-Bragado, M. Jaraiz, P. Castrillo, R. Pinacho, J. Barbolla, and M. M. de Souza, "Mobile silicon di-interstitial: Surface, self-interstitial clustering, and transient enhanced diffusion phenomena," Phys. Rev. B, vol. 68 , no. 19 , pp. $195204-1 / 5,2003$.

[17] Synopsys, Sentaurus reference Manual, 2007th ed., Mountain View, CA,USA, 2007.

[18] S. Takeda, "An atomic model of electron-irradiation-induced defects on $\{113\}$ in si," Jap. J. Appl. Phys., vol. 30, p. L639, 1991.

[19] F. Cristiano, J. Grisolia, B. Colombeau, M. Omri, B. de Mauduit, A. Claverie, L. F. Giles, and N. E. B. Cowern, "Formation energies and relative stability of perfect and faulted dislocation loops in silicon," J. Appl. Phys., vol. 87, no. 12, p. 8420, 2000.

[20] P. A. Stolk, H. J. Gossmann, D. J. Eaglesham, D. C. Jacobson, C. S. Rafferty, G. H. Gilmer, M. Jaraiz, J. M. Poate, H. S. Luftman, and T. E. Haynes, "Physical mechanism of transient enhanced dopant diffusion in ion-implanted silicon," J. Appl. Phys., vol. 81, pp. 6031-6050, 1997.

[21] K. Moller, K. S. Jones, and M. Law, "Cross-sectional transmission electron microscopy analysis of $\{311\}$ defects from si implantation into silicon," Appl. Phys. Lett., vol. 72, no. 20, pp. 2547-9, 1998. 